\title{
ABSTRACT THEORY OF PACKINGS AND COVERINGS. II by $\mathrm{S}$. SWIERCZKOWSKI \\ (Received 4th February, 1959)
}

1. Preliminaries and summary. The present paper is closely related to a paper with the same title by A. M. Macbeath [3]. We use many notions which are defined there for a measure-space; nevertheless we define them once more because we consider the slightly different case of a measure-ring.

Let $(\mathbf{S}, \mu)$ be a measure-ring with unity such that the measure $\mu$ is $\sigma$-finite (for definitions cf. [2]). We assume that there exists a countable group $G$ of transformations which map $\mathbf{S}$ onto itself and which preserve the measure $\mu$ and the operations $\checkmark,-, \cap$ in $\mathbf{S}$. We denote by $g A$ the image of $A \in \mathbf{S}$ by $g \in G$. For any subset $\Gamma$ of $G$ we write $\Gamma A$ instead of $\bigcup_{0 \in \Gamma} g A$. We say that an element $P$ belonging to $\mathbf{S}$ is a packing (more precisely, a $G$-packing) of an element $A \in \mathbf{S}$ if $g P \subset A$ for every $g \in G$ and the elements $g P$ are disjoint for different $g$. We call an element $C \in \mathbf{S}$ a covering of $A$ if $A \subset G C$. If an element $F \in \mathbf{S}$ is simultaneously a packing and a covering of $A$, then $F$ is called a fundamental domain for $A$. If, in particular, $A$ is the ring unity 1 , then we call $P$ (or $C$ ) a packing (or covering) of $\mathbf{S}$, and $F$ a fundamental domain for $\mathbf{S}$. In Theorem 1 we give a condition on $(S, \mu)$ and $G$ which is equivalent to the existence of a fundamental domain $F$ for $\mathbf{S}$.

If $P$ and $C$ are a packing and a covering of an element $A \in \mathrm{S}$, then $\mu P \leqslant \mu C$. This result is stated in [3] (Theorem 1) for the ring $\mathbf{S}$ of all measurable subsets of a measure-space $(X, S, \mu)$ and for $A=X$. However, the proof which is given there is more general and it can be applied to a measure-ring $(\mathbf{S}, \mu)$ and to an arbitrary $A \in \mathbf{S}$. We shall use this result in several parts of our proof, referring to it as to the theorem about packings and coverings.

Let $p$ be the upper bound of all measures $\mu P$, where $P$ are packings of $\mathbf{S}$, and let $c$ be the lower bound of measures $\mu C$, where $C$ are coverings of $\mathbf{S}$. These numbers exist since the zero element $0 \in \mathbf{S}$ is a packing and the ring unity $\mathbf{l}$ is a covering of $\mathbf{S}$. By the theorem about packings and coverings we have $p \leqslant c$. In Theorem 2 we give a condition on $(\mathbf{S}, \mu)$ and $G$ which is equivalent to $p=c$.

The corollaries contain results which are analogous to Theorems 1 and 2 but concern the ring of measurable sets of a measure-space. We construct also examples which show that these theorems fail to be true if the measure is not $\sigma$-finite.

2. Results. Let $e \in G$ be the identity transformation. We denote by $(\pi),(\rho)$ and $(\delta)$ the following properties :

( $\pi)$ If $A \in \mathbf{S}, g \in G, A \neq 0$ and $g \neq e$, then there exists a $B \subset A$ such that $B \neq 0$ and $B \cap g B=0$.

( $\rho)$ If $A \in \mathbf{S}$ has arbitrarily small coverings, then $A=0$.

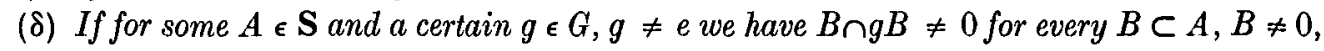
then $A$ has arbitrarily small coverings.

ThEorem 1. There exists a fundamental domain $\mathbf{F}$ for $\mathbf{S}$ if and only if both ( $\pi)$ and $(\rho)$ hold.

THEOREM 2. $p=c$ is equivalent to $(\delta)$. 
We shall verify in $\S 6$ that the $\sigma$-finiteness of $\mu$ is in both theorems an indispensable assumption. Let us consider now a measure-space $(X, S, \mu)$, where the measure $\mu$ is $\sigma$-finite and complete (see [2]). Let $G$ be a countable group of transformations of $X$ onto itself which preserve measurability and measure. We denote by $\left(\pi_{0}\right),\left(\pi_{0}^{\prime}\right),\left(\rho_{0}\right),\left(\delta_{0}\right)$ the properties :

$\left(\pi_{0}\right)$ If $A \in \mathbf{S}, g \in G, \mu A>0$ and $g \neq e$, then there exists $a B \subset A$ such that $\mu B>0$ and $B \cap g B=\phi$ ( $\phi$ is the empty set).

$\left(\pi_{0}^{\prime}\right)$ No $g \neq e$ has fixed points (i.e. $g x \neq x$ for $x \in X$ ).

( $\left.\rho_{0}\right)$ If $A \in \mathbf{S}$ has arbitrarily small coverings, then $\mu A=0$.

$\left(\delta_{0}\right)$ If for some $A \epsilon \mathbf{S}$ and a certain $g \in G, g \neq e$ we have $\mu(B \cap g B)>0$ for every $B \subset A$ with $\mu B>0$, then $A$ has arbitrarily small coverings.

Applying Theorems 1 and 2 to the measure-ring defined by $(X, \mathbf{S}, \mu)$, we obtain the corollaries :

COROLLARY 1. There exists a fundamental domain for $(X, \mathbf{S}, \mu)$ if and only if $\left(\pi_{0}\right),\left(\pi_{0}^{\prime}\right)$, $\left(\rho_{0}\right)$ hold simultaneously.

CoRollary 2. $p=c$ is equivalent to $\left(\delta_{0}\right)$.

Let us consider a locally compact and $\sigma$-compact topological group $H$. We denote by $\mu$ the Haar measure on $H$ and by $\mathbf{S}$ the ring of all $\mu$-measurable sets in $H$. Let $G$ be a countable subgroup of $H$. The left translations by elements of $G$ form a group of measure preserving transformations of the measure-space $(H, \mathbf{S}, \mu)$. Evidently $\left(\pi_{0}\right)$ holds. Hence $\left(\delta_{0}\right)$ is true and so $p=c$.

It follows from Corollary 1 that if $G$ is discrete (in the topology induced by $H$ ), then a fundamental domain exists. This is however a known result [1]. It follows also from Corollary 1 that if $G$ is not discrete, then no fundamental domain exists. But this can be proved also directly. In fact, a fundamental domain $F$ is of positive measure and we have $F \cap g F=\phi$ for $g \in G-\{e\}$. Thus $G-\{e\}$ cannot intersect every neighbourhood of $e$ (see [4]).

3. Two lemmas. Let us call coverings of $\mathbf{S}$ simply coverings and let us adopt the same convention for packings and fundamental domains.

Lемma 1. We assume that $(\pi)$ holds. Then every covering $C$ which is not a packing contains a covering $C_{0} \neq C$.

Proof. We have $C \cap g^{-1} C \neq 0$ for some $g \neq e$. Let $A=C \cap g^{-1} C$ and let $B \subset A$ satisfy


follows that $C_{0}=C-B$ is also a covering.

Lemma 2. If $(\pi)$ and $(\rho)$ hold and there exists a covering $C$ with $0<\mu C<\infty$, then a fundamental domain exists.

Proof. Let $\mathbf{C}$ be the family of all coverings of finite measure. We observe that a partial order is defined in $\mathbf{C}$ by the relation of inclusion. From Zorn's Lemma it follows that $\mathbf{C}$ contains a maximal decreasing chain $\mathbf{M}$, i.e. an ordered subfamily $\mathbf{M}$ of coverings such that no covering $C_{0} \in \mathbf{G}-\mathbf{M}$ is contained in all $C \in \mathbf{M}$. Let $a=\inf _{C \in \mathbf{M}} \mu C$. There exist coverings $C_{1}, C_{2}, \ldots, C_{n}, \ldots \in \mathbf{M}$ such that $a=\lim _{n \rightarrow \infty} \mu C_{n}$. Put $F=\bigcap_{n=1}^{\infty} C_{n}$. Thus $\mu F=a$. Let $B=G F$. Since $1=G C_{n}$ for each $n$ it follows that $1-B \subset G\left(C_{n}-F\right)$. We obtain from $\lim \mu\left(C_{n}-F\right)=0$ 
that $1-B$ has arbitrarily small coverings. Hence, by $(\rho)$, we have $B=1$; i.e. $F$ is a covering.

Let us verify that $F \subset C$ holds for every $C \in \mathbf{M}$. Indeed, from $F \cap C \neq F$ for some $C \in \mathbf{M}$ follows $\mu(F \cap C)<\mu F=a$, and thus $\mu \bigcap_{n=0}^{m} C_{n}<a$ for $C_{0}=C$ and sufficiently large $m$. This is a contradiction since $\bigcap_{n=0}^{m} C_{n} \in \mathbf{M}$. From $F \subset C$ for every $C \in \mathbf{M}$ we have that no covering $C_{0} \neq F$ is contained in $F$. Since $F$ is a covering, we obtain, by Lemma 1 , that $F$ is also a packing and thus $F$ is a fundamental domain.

4. Proof of Theorem 1. Suppose first that a fundamental domain $F$ exists. We shall prove that both $(\pi)$ and $(\rho)$ hold. Assume that $(\pi)$ is not true, i.e. that there exists an $A \in \mathbf{S}$ and $g \in G$ such that $A \neq 0, g \neq e$ and $B \cap g B \neq 0$ whenever $B \subset A$ and $B \neq 0$. From $A \subset G F$ we have that, for some $g_{0} \epsilon G$, the set $B=A \cap g_{0} F$ is not empty. From $B \subset A$ and $B \neq 0$ it follows that $B \cap g B \neq 0$. This is a contradiction, since $B \subset g_{0} F, g B \subset g g_{0} F$ and $g \neq e$.

Now suppose that $(\rho)$ is false. We assume that $A \neq 0$ has arbitrarily small coverings. It follows that the same is true for $G A$. Thus, by the theorem about packings and coverings, there exists no packing of $G A$ except 0 . Evidently $A \cap g_{0} F \neq 0$ for some $g_{0} \in G$. Thus $P=A \cap g_{0} F$ is a packing of $G A$ which is different from 0 and this is a contradiction.

Now let us suppose that $(\pi)$ and $(\rho)$ hold. We take a maximal set $\Phi$ of non-zero elements $A$ of finite measure such that all elements $G A(A \in \Phi)$ are disjoint. This set is countable since $\mu$ is $\sigma$-finite. Thus $\Phi=\left\{A_{1}, A_{2}, \ldots, A_{n}, \ldots\right\}$. Suppose that $\bigcup_{n=1}^{\infty} G A_{n} \neq 1$. By the $\sigma$-finiteness of $\mu$ the element $B=1-\bigcup_{n=1}^{\infty} G A_{n}$ contains an element $D \neq 0$ of finite measure. We have $G D \cap G A_{n}=0$ for every $n$ and this is a contradiction since $\Phi$ is maximal. Hence $1=\bigcup_{n=1}^{\infty} G A_{n}$. Since $A_{n}$ is a covering of $G A_{n}$, it follows from Lemma 2 that there exists a fundamental domain $F_{n}$ for each $G A_{n}$. Thus $F=\bigcup_{n=1}^{\infty} F_{n}$ is a fundamental domain for $\mathbf{S}$.

5. Proof of Theorem 2. We assume first that $(\delta)$ does not hold and we shall prove that then $p \neq c$. Let $A \in \mathbf{S}$ and $g \in G, g \neq e$ be such that for $B \subset A$ and $B \neq 0$ we have $B \cap g B \neq 0$, but $A$ does not have arbitrarily small coverings. It follows that the lower bound $m$ of measures of coverings of $G A$ is positive. Let us prove that every packing $P$ of $\mathbf{S}$ is disjoint from $G A$. Assume the contrary. Then $g_{1} A \cap g_{2} P \neq 0$ for some $g_{1}, g_{2} \in Q$ and thus $B=A \cap g_{1}^{-1} g_{2} P \neq 0$. We have $B \subset A, B \neq 0$ and thus it follows from $B \cap g B \neq 0$ that $g_{1}^{-1} g_{2} P \cap g g_{1}^{-1} g_{2} P \neq 0$. Therefore $P$ cannot be a packing. We now define $Q=1-G A$. If $C$ is an arbitrary covering of $\mathbf{S}$, then evidently $M=C \cap G A$ is a covering of $G A$ and $N=C \cap Q$ is a covering of $Q$. Consequently $\mu M \geqslant m$. We have $M \cup N=C, M \cap N=0$ and this implies $\mu N \leqslant \mu C-m$. Let $P$ be a packing of $S$. Since $P$ is disjoint from $G A, P$ is a packing of $Q$. Thus $\mu P \leqslant \mu N$, by the theorem about packings and coverings, and we obtain $\mu P \leqslant \mu C-m$. Therefore $p<c$ follows.

We assume now that $(\delta)$ holds and we shall prove that $p=c$. If $(\rho)$ holds, then $(\pi)$ follows by $(\delta)$, and then $p=c$ by Theorem 1. Suppose that $(\rho)$ does not hold and take a maximal set $\Omega$ of non-zero elements such that each $A \in \Omega$ has arbitrarily small coverings and the elements $G A$, where $A \in \Omega$, are disjoint. $\Omega$ is countable by the $\sigma$-finiteness of $\mu$ and 
thus also $Q=\bigcup_{A \in \Omega} G A$ has arbitrarily small coverings. We shall now prove that there exists a fundamental domain for $1-Q$. This follows from Theorem 1 . Indeed, $(\rho)$ holds for each $A \subset 1-Q$ by the construction of $Q$ and it remains to verify that $(\pi)$ holds also. But if $(\pi)$ is false for some $A \neq 0$ and $g \neq e$, then, by $(\delta), A$ has arbitrarily small coverings, contradicting $(\rho)$. Let $F$ be a fundamental domain for $1-Q$. For each $\varepsilon>0$ there exists a covering $D$ of $Q$ with $\mu D<\varepsilon$. It follows that $F \cup D$ is a covering and $F$ a packing of $S$. Thus $c \leqslant p$. By the theorem about packings and coverings, we have $p \leqslant c$ and therefore $p=c$.

6. Rings with a non $\sigma$-finite measure. We give first an example of a measure-ring $(\mathbf{S}, \mu)$ and a group $G$ such that $(\pi)$ and $(\rho)$ hold but no fundamental domain exists. Let $\mathbf{S}$ be the ring of all these sets of real numbers which either are countable or have a countable complement. Let $G$ be the group of translations by integers. The measure $\mu$ of $A \in \mathbf{S}$ is defined to be the number of elements in $A$ ( $\mu A$ is infinite if $A$ is infinite). Then $(\pi)$ and ( $\rho)$ hold. We observe that every packing of $\mathbf{S}$ is a countable set and every covering is not countable. Therefore no fundamental domain exists.

Now let us give an example where $(\delta)$ holds and $p \neq c$. Let $L$ be the ring of all Lebesguemeasurable sets of real numbers and let $N \subset L$ be the ideal of all sets of measure 0 . We denote by $L^{*}$ the quotient ring $L / N$. Let $T$ be an infinite non-countable set and let to each $\tau \in T$ correspond a replica $L_{\tau}^{*}$ of $L^{*}$. We consider the product $\mathbf{S}=\prod_{\tau \in T} L_{\tau}^{*}$. For $A \epsilon \mathbf{S}$ we denote by $A_{\tau}$ the $\tau$-coordinate of $A\left(A_{\tau} \in L_{\tau}^{*}\right)$. Let $m$ denote the Lebesgue measure in $L^{*}$. We define $\mu$ on $\mathbf{S}$ by

$$
\mu A=\sum_{\tau \in T} m A_{\tau},
$$

where the sum of a non-countable collection of positive numbers is defined to be infinite. For $A, B \in \mathrm{S}$ let $C=A \cup B$ if $C_{\tau}=A_{\tau} \cup B_{\tau}$ for every $\tau$. Similarly we define in $\mathbf{S}$ the operations - and $\cap$. Let $G$ be the group of translations of elements of $L$ by rational numbers. Thus for every $A \in \mathrm{S}$ and $g \in G$ we can define $g\left(A_{\tau}\right)$ for each $\tau$. Let us define $g A$ by $(g A)_{\tau}=g\left(A_{\tau}\right)$. Consequently $(\pi)$ is true and $(\delta)$ follows. Let us observe that if $P$ is a packing of $\mathrm{S}$, then each $P_{\tau}$ is a packing of $L_{\tau}{ }^{*}$ and thus $P_{\tau}=0$ by the theorem about packings and coverings. Hence 0 is the only packing of $\mathbf{S}$ and we have $p=0$. We easily observe that if $C$ is a covering of $\mathbf{S}$, then $\mu C=\infty$. Therefore $p \neq c$.

7. Proofs of the corollaries. Let $N$ be the ideal of all subsets of $X$ which are of measure 0 ; these sets form an ideal since the measure $\mu$ is complete. We consider the measurering $\left(\mathbf{S}^{*}, \mu\right)$, where $\mathbf{S}^{*}$ is the quotient ring $S / N$. Let us denote by $A^{*} \in \mathrm{S}^{*}$ the image of $A \in \mathbf{S}$ by the natural mapping of $\mathbf{S}$ onto $\mathbf{S}^{*}$.

We first prove Corollary 1. Suppose that $\left(\pi_{0}\right),\left(\pi_{0}^{\prime}\right)$ and $\left(\rho_{0}\right)$ hold. Then $(\pi)$ and $(\rho)$ hold for $\mathrm{S}^{*}$. Thus, by Theorem 1, there exists an $F \in \mathbf{S}$ such that $F^{*}$ is a fundamental domain for $S^{*}$. It follows that

$$
P=F-(G-\{e\}) F
$$

is a packing of $\mathbf{S}$ such that $Q=X-G P \in N$. Evidently $Q$ is a union of sets $G x$ where $x \in Q$. Let $D \subset Q$ be any set which contains exactly one element from each of these sets $G x$. We have $D \in N$, and thus $D$ is measurable. It follows from $\left(\pi_{0}^{\prime}\right)$ that $D$ is a fundamental domain for $Q$. Consequently $P \cup D$ is a fundamental domain for $\mathbf{S}$. 
Conversely, if $F$ is a fundamental domain for $\mathbf{S}$, then evidently $F^{*}$ is a fundamental domain for $\mathbf{S}^{*}$ and the necessity of $\left(\pi_{0}\right)$ and $\left(\rho_{0}\right)$ follows. The necessity of $\left(\pi_{0}^{\prime}\right)$ is obvious.

To prove Corollary 2 it suffices to observe that to every packing of $\mathbf{S}$ corresponds a packing of $\mathbf{S}^{*}$ of the same measure and conversely, and that the same is true for coverings.

\section{REFERENCES}

1. C. Chabauty, Limite d'ensembles et géométrie des nombres, Bull. Soc. Math. France 78 (1950), 143-151.

2. P. R. Halmos, Measure theory (New York, 1951).

3. A. M. Macbeath, Abstract theory of packings and coverings I, Proc. Glasgow Math. Assoc. 4 (1959), 92-95.

4. A. Weil, L'integration dans les groupes topologiques et ses applications (Paris, 1953), p. 50.

Qdeen's College

DUNDEE 